\title{
Endoscopic Foraminotomy in Spinal Epidural Lipomatosis, Case Report
}

\author{
Diego Quillo-Olvera ${ }^{1}$, MD, Jin Sung Kim ${ }^{2}$, MD, Javier Quillo-Olvera ${ }^{3}$ MD, Javier Quillo-Resendiz ${ }^{3}$ MD \\ ${ }^{1}$ Department of Neurosurgery, The Regional Hospital “Dr Valentin Gomez Farias” ISSSTE. Guadalajara Jalisco, Mexico \\ ${ }^{2}$ Neurosurgery and Spine surgery, Department of Neurosurgery, The Catholic University of Korea, \\ Seoul St. Mary's Hospital, 222, Banpo-daero, Seocho-gu, Seoul, South Korea \\ ${ }^{3}$ Neurosurgery and Spine surgery, The Brain and Spine Care Center, Minimally Invasive Spine Surgery Center, \\ Querétaro City, México
}

*Corresponding Author: Diego Quillo-Olvera, MD; drquilloolvera86@gmail.com

Postal Address: Doctores \# 102 colonia el Marques, Santiago de Querétaro CP 76047, Mexico

Received 07 May 2019;

Accepted 21 June 2019;

Published 30 June 2019

\begin{abstract}
A 69-year-old woman with a history of hypertension and Cushing's syndrome, Her symptoms onset a year ago with pain in both legs treated with analgesics, but continued with right L4-L5 radiculopathy, she underwent treatment with endoscopy lumbar transforaminal, relieving his discomfort in the right leg, indicating on the visual analog scale (VAS) a score of $2 / 10$. Four months later she started with pain in the left leg pointing to VAS of 7/10 in the disposition of the roots of L4-L5. Magnetic resonance imaging (MRI) of lumbar spine in sagittal and axial sequence weighted in T1 and T2 showed an excess of epidural fat and compression of the dural sac from L1 to S1. After the clinical-radiological assessment, the patient was treated with a left L4-L5 endoscopic foraminotomy in which adipose tissue was obtained and a decompression of the neural structures was achieved, the pain and weakness of the leg improved, indicated a VAS of 2/10 and the MacNab criteria scale for clinical graduation reported as good.

We present a case of spinal epidural lipomatosis caused by Cushing's syndrome in a patient who underwent a lumbar endoscopic foraminotomy for radicular pain in a hospital in Korea.
\end{abstract}

Keywords: spinal epidural lipomatosis; Cushing syndrome; lumbar endoscopic foraminotomy; epidural fat.

\section{Introduction}

In 1975, Lee described the extrinsic compression of the spinal cord in a patient treated with steroids as a treatment after a kidney transplant ${ }^{[1]}$. Epidural lipomatosis is the proliferation of fat in the epidural space that results in the compression of the cord medullar causing nerve deficit, the thoracic and lumbar regions are the most frequent sites of affection ${ }^{[2]}$. Endogenous and exogenous causes of excess glucocorticoids such as glucocorticoid injections, hypothyroidism, obesity and other idiopathic factors have been associated with the development of spinal epidural lipomatosis, the symptoms depend on the severity of spinal compression such as non-specific back pain, radiculopathies and medullar syndrome. The diagnosis can be made by CT scanning and MRI. Treatment ranges from withdraw steroids from treatment, weight loss to spine surgery ${ }^{[3,4]}$.

We present a case of spinal epidural lipomatosis caused by Cushing's syndrome in a patient who underwent a lumbar endoscopic foraminotomy for radicular pain in a hospital in Korea.

\section{Case report}

A 69 year old female, past medical history included hypertension, diabetes, cushingoid appearance moon facies, buffanlo hump, generalized obesity, transphenoidal surgical approach for pituitary granular cell tumor correspondent to a adrenocorticotropic adenoma and several vertebroplasty include T8,T12,L1,L4 and L5 for osteoporosis. Her symptoms onset a year ago with both leg pain managed with conservative treatment, but she had persistent sciatica in the distribution of the right L4-L5 nerve root. In January 2018 she underwent a right side endoscopic transforaminal approach L4-L5. She reported to recover from her leg right pain immediately after the operation with visual analogue scale (VAS) for the right leg was $2 / 10$, four months later she complains of contralateral leg pain in same level with VAS 7/10.

T2 - weighted sagittal and T1 - weighted axial sequence magnetic resonance imaging (Fig.1) showed extensive epidural fat with dural sac compression from L1 to S1. A left L4-L5 endoscopic foraminotomy were the fat tissue was removed and decompress until observe free floating neural structures, postop computed 
tomography showed the path of the foraminotomy in the superior and inferior facet (Fig.2). The pain and weakness of the leg improved after the surgery outcomes were reported VAS $2 / 10$ and $\mathrm{McNab}$ criteria as good.

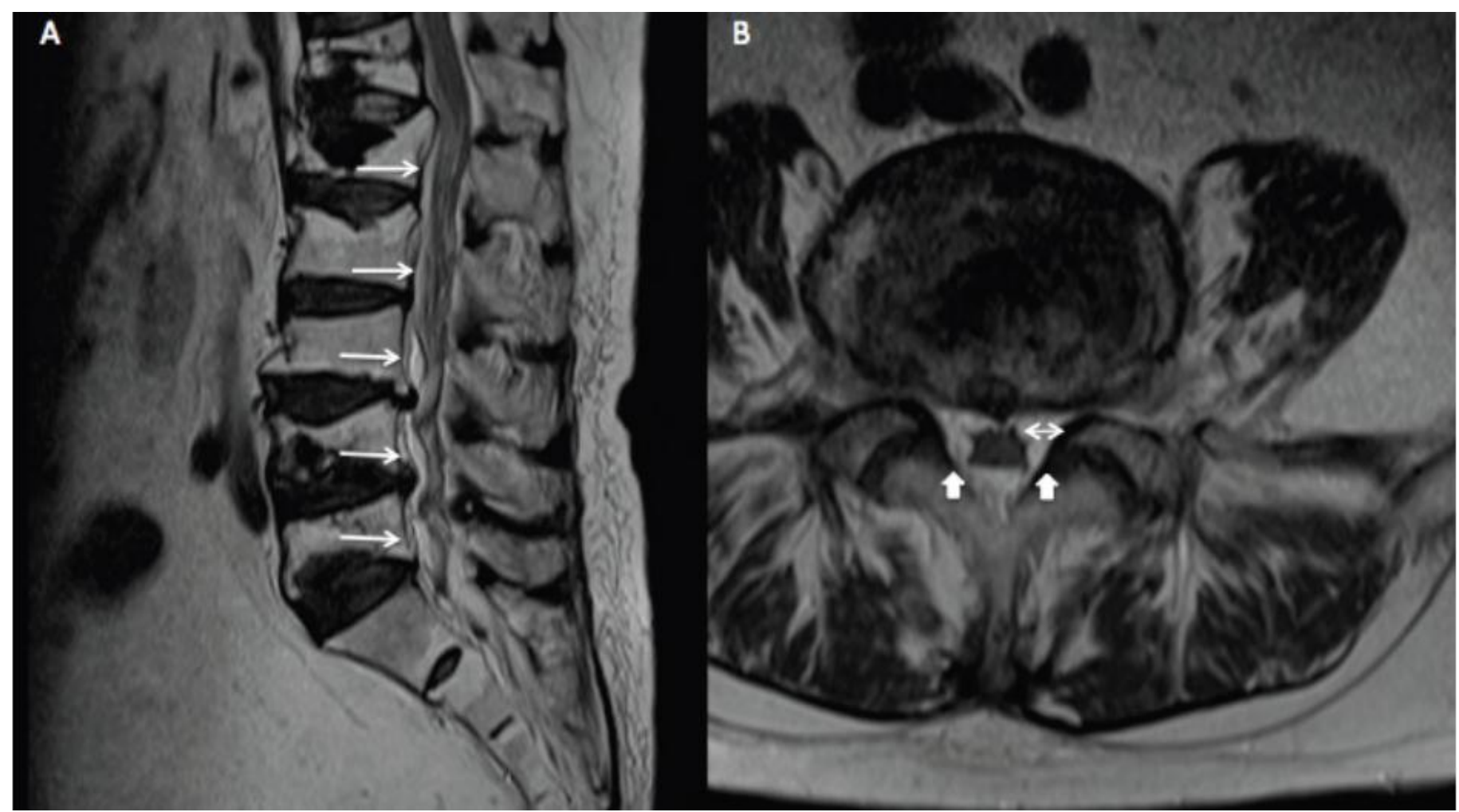

Fig.1: Lumbar sagittal and axial magnetic resonance imaging.AT2-weighted sagittal, B T1-weighted axial image. Thin arrows indicate the extensive epidural compression from L1 to S1, thickarrows mark the circumferential epidural fat with dural sac compression.

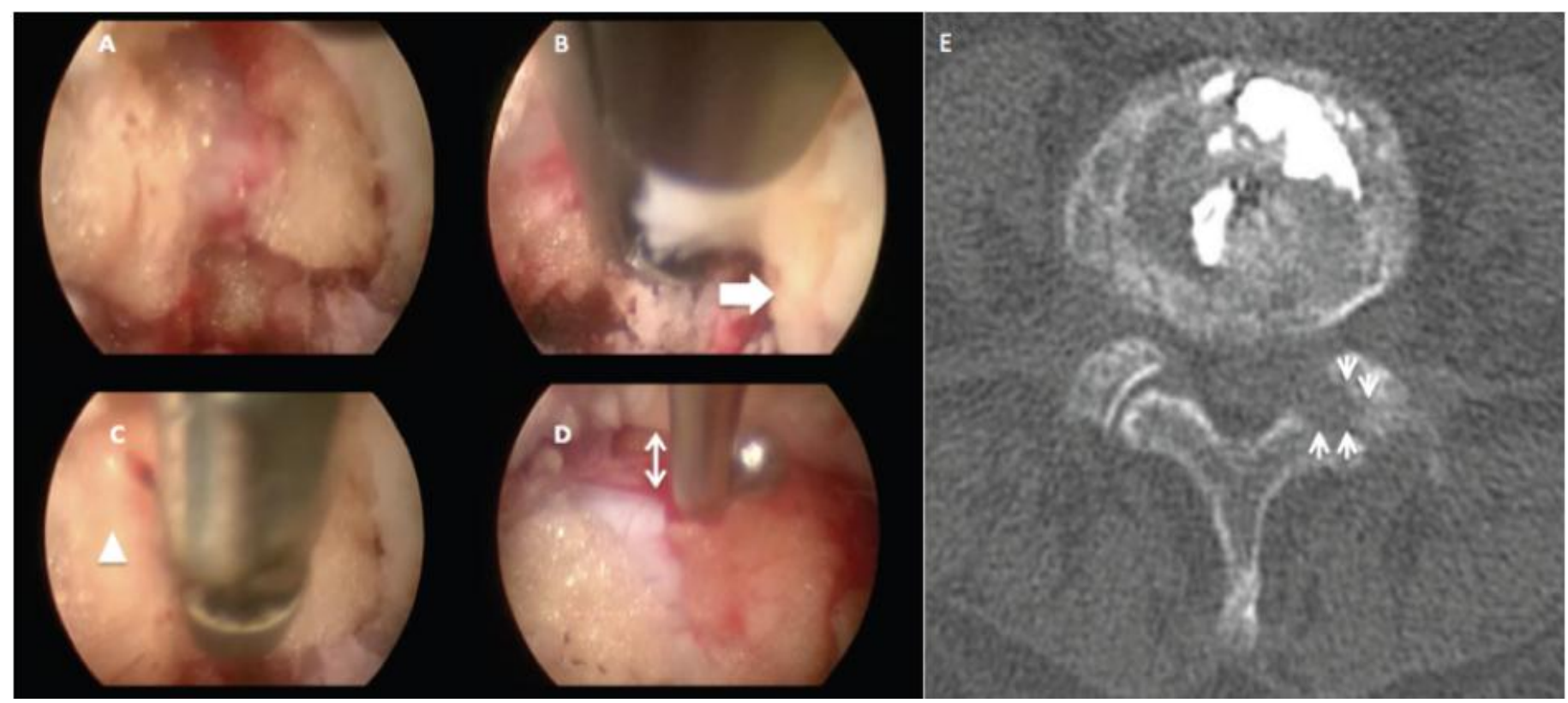

Fig.2: Intraoperative endoscopic View. A transforaminal view show the epidural fat surrounds the neural elements, B endoscopic tool remove lateral flavum (thick arrow), $\mathrm{C}$ fat ablation (triangle) with endoscopic radiofrequency, $\mathrm{D}$ neural elements released with free floating space(opposite arrows).E Lumbar CT L4 axial view, arrows shows path of the left foraminotomy

\section{Discussion}

The spinal epidural lipomatosis is a rare condition wich pathophysiological mechanismis consists of excessive fat acumulation in epidural space causes dural sac and nerve root compression that leads to neurological symptoms ${ }^{[5]}$.

Long-term administration of exogenous glucocorticoids equivalent to $30-100 \mathrm{mg}$ of prednisone per day is known as the first cause for SEL, other endogenous causes such as Cushing syndrome, hypothyroidism, pituitary tumors, obesity have been identified for the generation of excess epidural fat $^{[6]}$, the average age of presentation of the disease is 43 years and most commonly affects men at the level of the thoracic and lumbar spine, at the histological level there is no difference between the hypertrophy of the fatty tissue in spinal lipomatosis and the subcutaneous tissue $^{[7,8]}$.

All the studies reported to date describe a slow progression of the clinical manifestations and depend on the site of localization, they 
are mentioned as non-specific back pain with muscular weakness, radiculopathy or cauda equina syndrome ${ }^{[9]}$, the tomography show a low density of fatty tissue but it is not possible to differentiate it from the anatomical structures within the spinal canal, on the other hand in the MRI the epidural fat is hyperintense in T1 and T2 with the compression of the spinal cord at the thoracic level and the dural sac at lumbosacral level, the sign of the "Y" transversal section is observed compression not only of the dural sac but also of the exit and traversing nerve roots ${ }^{[10]}$.

The treatment of spinal epidural lipomatosis includes the suspension of steroids, restriction of fats in the diet or surgery, since it is required to remove epidural compression despite conservative measures $^{[2]}$. Currently, the minimally invasive spine surgery is used to reduce the short and long-term complications associated with conventional open-ended spinal surgery, obtaining the same decompression outcomes similar to conventional spinal surgery and endoscopy is the worldwide novelty in these techniques $^{[11]}$.

In the transforaminal endoscopy approach there is no destabilization and no access related morbidity but we can see inside of the disc well, offers the greatest and most flexible access to the lumbar disc without the need for laminectomy and this access avoids excessive removal of ligamentum flavum and that is significant in preventing scar formation ${ }^{[12]}$.

We did a search in PubMed reviews between the relationship of spine epidural lipomatosis with Cushing syndrome finding only 6 items since1992 until 2004, in these works conservative treatment, removing the tumor that caused hypercotisolism and decompressive laminectomy were used to release the compression of the spinal cord and subsequent symptoms. That is why the incidence of this disease in association with endogenous ${ }^{[13-15]}$.

In this case the patient was managed by an transforaminal endoscopy approach for the unilateral radiculopathy that improved the symptoms after removal of the fat that was causing a compression of the neural structures. There's no studies about endoscopic surgery procedure in treating patientes with lipomatosis.

\section{Conclusions}

We propose a safe and feasible endoscopic approach for unilateral radiculopathies presented by patients with SEL, we consider that this case is interesting because there are very few works published to date, however, endoscopic procedures require a learning curve and it must be taken into account that complications can occur, therefore it is not a harmless procedure but in experienced hands excellent results can be obtained as it was in this case.

\section{References}

[1] Lee M, Lekias J, Gubbay SS, Hurst PE. Spinal cord compression by extradural fat after renal transplantation. Med J Aust 1975: 1: 201-3.

[2] B. Fiirgaard, F. H. Madsen. Spinal epidural lipomatosis. Case report and review of the literatura. Scand J Med Sci Sports 1997: 7: 354-357.

[3] Al-Khawaja D, Seex K, Eslick GD. Spinal epidural lipomatosis a brief review.J Clin Neurosci. 2008:12:1323-6.

[4] Kurt E, Bakker-Niezen SH. Neurogenic claudication by epidural lipomatosis: a case report and review of literature. Clin Neurol Neurosurg.1995: 4:354-357

[5] Fogel GR, Cunningham PY 3rd, Esses SI: Spinal epidural lipomatosis: case reports, literature review and meta-analysis. Spine J 5: 202-211, 2005

[6] Haddad SF, Hitchon PW, Godersky JC. Idiopathic and glucocorticoid-induced spinal epidural lipomatosis. Journal of neurosurgery. 1991; 74: 38-42.

[7] Fessler RG, Johnson DL, Brown FD, Erickson RK, Reid $\mathrm{SA}$, et al. Epidural lipomatosis in steroid-treated patients. Spine. 1992; 17: 183-188.

[8] Fassett DR, Schmidt MH. Spinal epidural lipomaᄀtosis: a review of its causes and recommendations for treatment. Neurosurgical focus. 2004; 16: E11.

[9] Lisai P, Doria C, Crissantu L, Meloni GB, Conti M, et al. Cauda equina syndrome secondary to idiopathic spinal epidural lipomatosis. Spine. 2001; 26: 307-309.

[10] Kuhn MJ, Youssef HT, Swan TL, Swenson LC. Lumbar epidural lipomatosis: the "Y" sign of thecal sac compression. Computerized medical imaging and graphics: the official journal of the Computerized Medical Imaging Society. 1994; 18: 367-372.

[11] Choi G, Pophale CS, Patel B, Uniyal P. Endoscopic spine surgery. J Korean Neurosurg Soc 60 (5): 485-497, 2017

[12] Gore S, Yeung A. The "inside out" transforaminal technique to treat lumbar spinal pain in an awake and aware patient under local anesthesia: results and a review of the literature. Int J Spine Surg. 2014;8:28.

[13] Bodelier AG, Groeneveld W, van der Linden AN, Haak HR. Symptomatic epidural lipomatosis in ectopic Cushing's syndrome, Eur J Endocrinol. 2004 Dec;151(6):765-9.

[14] Koch CA, Doppman JL, Watson JC, Patronas NJ \& Nieman LK. Spinal epidural lipomatosis in patient with the ectopic cortico- tropin syndrome. New England Journal of Medicine 19993411399 - 1400.

[15] Sivakamur K, Sheinart K, Lidov M \& Cohen B. Symptomatic spinal epidural lipomatosis in a patient with Cushing's disease. Neurology 1995452281 - 2283. 